\title{
Neuropathic alterations of the myenteric plexus neurons following subacute intraperitoneal administration of salsolinol
}

\author{
Magdalena Kurnik ${ }^{1}$, Krzysztof Gil ${ }^{1}$, Mariusz Gajda ${ }^{2}$, Piotr Thor ${ }^{1}$, Andrzej Bugajski ${ }^{1}$ \\ ${ }^{1}$ Department of Pathophysiology, Jagiellonian University Medical College, Krakow, Poland \\ ${ }^{2}$ Department of Histology, Jagiellonian University Medical College, Krakow, Poland
}

\begin{abstract}
Introduction. Impairment of the enteric nervous system has been suggested to occur within the pathogenesis of neurodegenerative diseases. Thus, in the current study, we consider salsolinol (1-methyl-6,7-dihydroxy-1,2,3,4-tetrahydroisoquinoline, SAL) as a substance that can potentially induce myenteric neurodegeneration.

Material and methods. Male Wistar rats were subjected to continuous intraperitoneal dosing of salsolinol ( $200 \mathrm{mg} / \mathrm{kg}$ in total) with osmotic mini-pumps for either two or four weeks. An equivalent group of rats served as the control. Jejunal myenteric neurons were subjected to immunofluorescence staining to detect neuron specific protein - protein gene product (pan-neuronal marker, PGP 9.5), nitric oxide synthase (NOS), choline acetyltransferase (ChAT), Bax-protein and alpha-synuclein. In search of any functional impairment within the gastrointestinal tract, gut motility was assessed by determining the residual solid food contents in the stomach and the small and large intestine transit.

Results. The myenteric neuron count, the mean size of the neuron body, the area of ganglia and the diameter of nerve strands were decreased in both of the salsolinol-treated groups compared with the controls. The number of NOS-positive cells was lower in the salsolinol-treated groups, while the number of ChAT-positive cells remained unchanged in comparison with the controls. Neurons expressing the pro-apoptotic Bax protein and alpha-synuclein deposits were observed among the myenteric neurons of the salsolinol-treated rats.

Conclusions. Salsolinol evokes enteric neuronal cell death via initiation of apoptosis and leads to the formation of pathological aggregates of alpha-synuclein. Impairment of myenteric neurons, mainly the inhibitory motor neurons, might be responsible for the abnormal intestinal transit. Thus, salsolinol might be regarded as a suitable compound for inducing experimental enteric neurodegeneration in rats. (Folia Histochemica et Cytobiologica 2015, Vol. 53, No. 1, 49-61)
\end{abstract}

Key words: salsolinol; neurodegeneration; myenteric plexus; LMMP; NOS; ChAT; alpha-synuclein; PGP 9.5; rat

\section{Introduction}

The enteric nervous system (ENS) is a specific division of the autonomic nervous system and appears to be distinctive due to its intrinsic ability to mediate reflex

\footnotetext{
Correspondence address: M. Kurnik, Ph.D., M.Sc.

Department of Pathophysiology, Jagiellonian University Medical College

Czysta St. 18, 31-121 Krakow

tel.: + 4812 6333947, fax: +48126329056

e-mail:magdalena.kurnik@uj.edu.pl
}

activity, even when disconnected from the central circuits. ENS is responsible for main patterns of gut behavior, including motility, blood flow, secretion and absorption [1,2]. Exposure to stress, which is defined as an acute threat to homeostasis, results in alterations of the brain-gut axis ultimately leading to the development of a broad array of gastrointestinal disorders [3]. Enteric failure is also thought to be involved in the pathophysiology of Parkinson's disease (PD). For example, disturbances of gastrointestinal function observed in those patients, such as constipation and delayed gastric emptying, detract immensely from 
quality of life, even though do not pose an imminent life-threatening situation. Epidemiological evidence has shown that gut dysfunctions even predate well-defined motor symptoms [4-7]. Braak et al. proposed that the pathological staging of the disease should be directly linked to its clinical progression. According to their theory, environmental factors are able to cross the intestinal barrier, initiate the process of enteric neurodegeneration and then trigger its progression into the central nervous system [8-11]. However, it remains unclear how exactly such pathological processes are initiated and promoted.

Different toxins, such as 6-OHDA (6-hydroksydopamine), MPTP (1-methyl-4-phenyl-1,2,3,6-tetrahydropyridine), paraquat, maneb and rotenone (three commonly used pesticides), are often used to generate enteric Parkinsonian-like neuronal degeneration [12-16]. Morphological and quantitative alternations (especially the presence of pathological alpha-synuclein aggregates) in the ENS neurons, together with gut dysmotility, malabsorption and disrupted excretion, have been observed after the animals were subjected to those different neurotoxins. However, the pace of development of new animal models of Parkinson's disease is still prevailing due to their flaws as well as difficulty to produce a model that completely incorporates the underlying pathological process, which is still imperfectly understood in PD [14, 17]. Salsolinol (1-methyl-6,7-dihydroxy-1,2,3,4-tetrahydroisoquinoline) might represent such a neurotoxic candidate. It can be easily endogenously synthesized from dopamine and acetaldehyde by salsolinol synthase or from dopamine and pyruvic acid or delivered exogenously [18-20].

The aim of our study was to evaluate the effects of exogenous salsolinol on the morphology and morphometry of myenteric neurons in rat intestine and assess the related functional changes in the gastrointestinal tract of rats. The obtained results suggest that salsolinol might be regarded as an interesting substance for inducing experimental enteric neurodegeneration in rats.

\section{Material and methods}

Animals. Male albino Wistar rats (Jagiellonian University Medical College Animal Laboratory, Krakow, Poland), weighing $230-265 \mathrm{~g}$ at the beginning of the treatments, were individually housed in cages at a constant temperature of $22-24^{\circ} \mathrm{C}$ under a 12:12 hour light/dark cycle. Solid chow (Labofeed, Kcynia, Poland) and tap water were accessible ad libitum. All animal experiments were approved by the Jagiellonian University Bioethical Committee (protocol number $-67 / 2009,25^{\text {th }}$ June 2009) and conducted in accordance with Good Laboratory Practices.
Gross behavior. The general health status and motor function of the experimental animals were evaluated daily by observing their in-cage behavior and during handling to look for any signs of dehydration, abnormal thermoregulation, rigidity, bradykinesia or akinesia, dystonia or piloerection [21].

Experimental protocol. The rats were either subjected to continuous dosing with racemic salsolinol or served as controls. Salsolinol (salsolinol hydrochloride, Sigma, USA), at a total dose of $200 \mathrm{mg} / \mathrm{kg}$, was dissolved in $200 \mu \mathrm{L}$ of $0.9 \%$ sodium chloride solution (Baxter, Warsaw, Poland) and delivered via osmotic mini-pumps (ALZET, Durect Corporation, Cupertino, CA, USA) that were implanted intraperitoneally. The mini-pumps were implanted under sodium pentobarbital anesthesia $(25 \mathrm{mg} / \mathrm{kg}$, administered intraperitoneally, Vetbutal, Biowet, Pulawy, Poland). One group (designated the Salsolinol 1 group, S1) received salsolinol for two weeks (osmotic mini-pump model 2002) at a delivery rate of $0.5 \mu \mathrm{L} / \mathrm{h}(0.250 \mathrm{mg}$ of salsolinol per hour $)$, while the other (Salsolinol 2 group, S2) received salsolinol for four weeks (osmotic mini-pump model 2004) at a delivery rate of $0.25 \mu \mathrm{L} / \mathrm{h}(0.125 \mathrm{mg}$ of salsolinol per hour); all rats in both of the salsolinol-treated groups received the same cumulative amount of the drug. Control rats received vehicle in the morphological study group (C) and the functional study groups ( $\mathrm{C} 1$ and $\mathrm{C} 2)$.

According to both Fornai et al. [22] and Alvarez-Fisher et al. [23] only continuous intraperitoneal administration of a neurotoxin with an osmotic mini-pump may induce a neurodegenerative state in animals that mimics features of human disease, in much better way than an acute bolus injection or an intraperitoneal injection and results in lower acute toxicity.

At the end of the treatment period (day 15 or day 29), the rats were euthanized via decapitation, and fragments of the jejunum were removed for the morphological assessment of myenteric neurons.

Functional tests. One-hour stool output in response to a novel environment. The one-hour stool frequency was measured once a week after the initiation of salsolinol infusion. The assays were performed between 10:00 am and 12:00 pm. Each animal was removed from its home cage and placed in a clean, clear plastic cage without food or water for $1 \mathrm{~h}$. Stool specimens were transferred to sealed tubes immediately after expulsion. The total stool sample was weighed to obtain the wet weight, then dried overnight at $65^{\circ} \mathrm{C}$ and weighed again to obtain the dry weight. The stool water content was then calculated as an indicator of colon water absorption. The results were normalized to body weight (adapted from a method described in Li et al. and Greene et al. [24, 25]).

Determination of residual solid food in the stomach and gastric emptying. Following overnight fasting, the rats were allowed 
free access to pre-weighed food for sixty minutes. Thirty minutes later, the animals were euthanized via decapitation, and the stomach was excised and weighed, then dried using filter paper after removal of its contents and weighed again. The amount of food in the stomach was calculated as the difference between the total weight of the stomach with its contents and the weight of the stomach wall after the contents were removed, and subsequently dried (adapted from a method described in $\mathrm{Li}$ et al., Greene et al. and Anitha et al. [24-26]). The food pellets were weighed before and after the feeding period to determine the amount consumed by each animal and adjusted to body mass of animals ( $\mathrm{g}$ per $1 \mathrm{~kg}$ of body mass). The residual solid food in the stomach after 90 minutes was determined according to the following equation: $\%$ of residual solid food in the stomach $=$ gastric content/food intake $\times 100$. The gastric emptying during 90 minutes was determined according to the following equation: $\%$ of gastric emptying $=(1-$ gastric content $/$ food intake) $\times 100$. The water content was calculated as the difference between the wet and dried stomach content $(\%)$.

Measurement of small intestine transit. Following overnight fasting, the rats were allowed free access to specially prepared food, consisting of a mixture of high-fat food (Bento-Kronen, Versele-Laga, Deinze, Belgium) and charcoal (10:1), for $1 \mathrm{~h}$. Thirty minutes later, the animals were euthanized via decapitation, and the small intestine was removed. The whole length of the small intestine (duodenum, jejunum and ileum) was divided into ten equal parts, and the presence of charcoal was recorded macroscopically. The distance that the undigested food had travelled was taken as an estimate of transit in the small intestine. The transit was expressed as a percentage, with the first part of the bowel corresponding to $10 \%$ transit and the last part of the bowel to $100 \%$ transit.

Whole-mount longitudinal muscle-myenteric plexus (LMMP) preparations. Whole-mount preparations were produced to study the morphology of rats' myenteric plexus. Briefly, pieces of the proximal part of the small intestine (jejunum) were opened via incision along the mesenteric attachment, then pinned and stretched slightly on a Petri dish layered with Sylgard 184 (Silicone Elastomer Kit, Dow Corning, Midland, MI, USA) with the mucosal surface facing up and subsequently washed in 3 changes of $0.1 \mathrm{M}$ phosphate-buffered saline (PBS; $\mathrm{pH}$ 7.4). The tissue was then fixed by immersion in Zamboni's fixative [27] overnight at $4^{\circ} \mathrm{C}$. Following a brief rinse in PBS, the tissue was washed vigorously at room temperature in several changes of $50 \%$ ethanol in distilled water. The removal of picric acid was complete after 3 to 5 changes (10 min each) of ethanol. The tissue was then washed in 3 to 5 changes (10 min each) of PBS.

The mucosa, submucosa and circular muscle were then removed to facilitate the penetration of antibodies to the myenteric plexus under the S4E stereomicroscope (Leica, Wetzlar, Germany) with an Intralux 5100 light source (Volpi AG, Schlieren, Switzerland) using appropriate clamps and fine scissors. Whole-mount preparations containing longitudinal muscle and myenteric plexus (LMMP) were rinsed in PBS and cut into pieces of approximately $5 \mathrm{~mm} \times 5 \mathrm{~mm}$. A heat-induced antigen retrieval protocol was applied to unmask antigen determinants by immersion of the slides (free floating) in a water bath at $90-95^{\circ} \mathrm{C}$ in DAKO Target Retrieval Solution (Dako, Glostrup, Denmark) for $20 \mathrm{~min}$.

Immunofluorescence. Whole-mount preparations were incubated for $30 \mathrm{~min}$ in PBS with appropriate normal serum and 0.3\% Triton X-100 (Sigma-Aldrich, St. Louis, MO, USA) at room temperature, followed by overnight incubation at $4^{\circ} \mathrm{C}$ in a solution of PBS with appropriate normal serum containing the primary antibody and $0.3 \%$ Triton $\mathrm{X}-100$. Following 5 washes (10 min each) in PBS, the whole mounts were incubated for $1 \mathrm{~h}$ at room temperature with the secondary antibody diluted in PBS with appropriate normal serum and $0.3 \%$ Triton X-100. Finally, the whole-mount preparations were washed in two changes (10 min each) of PBS and mounted on gelatin-coated slides, air-dried, and cover-slipped with UltraCruz Mounting Medium (Santa Cruz Biotechnology, Dallas, TX, USA). Afterwards, the labeled whole mounts were analyzed immediately.

Antisera for immunofluorescence. The following primary antisera were used (Table 1): anti-PGP 9.5 (pan-neuronal marker - protein gene product, Dako); anti-NOS (nitric oxide synthase, Santa Cruz Biotechnology); anti-ChAT (choline acetyltransferase, Merck Millipore, Darmstadt, Germany); anti-TH (tyrosine hydroxylase, Millipore); anti-TH (tyrosine hydroxylase, Santa Cruz Biotechnology); and anti-alpha-synuclein (BD Bioscience, San Jose, CA, USA).

The following secondary antibodies were used (Table 1): polyclonal donkey anti-goat/FITC (Santa Cruz Biotechnology); FITC-conjugated polyclonal swine anti-rabbit (Dako); RPE-conjugated polyclonal goat anti-mouse (Dako); Cy3-conjugated polyclonal goat anti-rabbit (Jackson, West Grove, PA, USA); and biotinylated goat anti-mouse together with FITC-conjugated streptavidin (Jackson). The quality of Jackson's secondary antibodies used in the experiment was already extensively and successfully examined [28].

Detection of the Bax protein. To visualize apoptosis, jejunal tissue fragments were fixed in formalin ( $8 \%$ formaldehyde in $0.1 \mathrm{M}$ phosphate buffer, $\mathrm{pH} 7.4$ ), and paraffin-embedded $5 \mu \mathrm{m}$ slices were prepared. A heat-induced antigen retrieval protocol was applied to unmask antigen determinants via immersion of the slides in a water bath at $90-95^{\circ} \mathrm{C}$ in DAKO Target Retrieval Solution for $20 \mathrm{~min}$. The sections were first pre-treated with Protein Block Serum Free (Dako) and $0.3 \%$ Triton $\mathrm{X}-100$ at room temperature for $30 \mathrm{~min}$ to 
Table 1. Types, sources and dilution of antibodies

\begin{tabular}{|l|c|c|}
\hline Antibody & Catalog number and company & Dilution \\
\hline \multicolumn{2}{|c|}{ Primary antibodies } & $1: 200$ \\
\hline Polyclonal rabbit anti-PGP 9.5 & Z5116, Dako & $1: 100$ \\
\hline Polyclonal goat anti-NOS & sc-49055, Santa Cruz & $1: 150$ \\
\hline Polyclonal rabbit anti-ChAT & AB143, Millipore & $1: 100$ \\
\hline Monoclonal mouse anti-TH & AB318, Millipore & $1: 150$ \\
\hline Monoclonal mouse anti-TH & sc-47708, Santa Cruz & $1: 200$ \\
\hline Polyclonal rabbit anti-c-Kit & A4502, Dako & $1: 500$ \\
\hline Polyclonal rabbit anti-Bax & PC66, Calbiochem & $1: 40$ \\
\hline Monoclonal mouse anti- $\alpha$-synuclein & 610787, BD Bioscience & $1: 100$ \\
\hline & Secondary antibodies & $1: 40$ \\
\hline FITC-conjugated polyclonal donkey anti-goat & J2609, Santa Cruz & $1: 500$ \\
\hline FITC-conjugated polyclonal swine anti-rabbit & F0205, Dako & $1: 400$ \\
\hline RPE-conjugated polyclonal goat anti-mouse & R0480, Dako & $1: 500$ \\
\hline Cy3-conjugated polyclonal goat anti-rabbit & $111-165-144$, Jackson & \\
\hline FITC-conjugated streptavidin & $016-010-084$, Jackson & \\
\hline Biotinylated goat anti-mouse & $115-065-146$, Jackson & \\
\hline
\end{tabular}

block non-specific binding, and then incubated overnight at $4^{\circ} \mathrm{C}$ with a primary polyclonal rabbit anti-Bax antibody (Calbiochem/Merck Millipore, Darmstadt, Germany) in DAKO Dilution Solution (Dako). Following incubation with the primary antibody, the sections were washed with $0.1 \mathrm{M}$ phosphate buffer and processed using the LSAB2 (Dako) visualization kit with 3,3'-diaminobenzidine (DAB, Dako) as a chromogen, according to the manufacturer's instructions. The specimens were cover-slipped with glycerol and analyzed immediately.

Image analysis. All images were obtained using either an AXIOPHOT light microscope (Zeiss, Jena, Germany) or a JENAMED 2 fluorescence microscope (Zeiss, Jena, Germany) equipped with a ProgRes C12 plus digital camcorder (Jenoptik, Jena, Germany). Only the anti-mesenteric aspect of the jejunum was sampled. Digital images were collected at $250 \times$ magnification and quantified using computer-based image analysis system Multiscan 18.03 software (CSS, Warsaw, Poland). In order to calibrate the software (semi-automatic measurement), the Bürker chamber was placed on the microscope stage and the counting grid was brought into focus. The measured area of the microscopic field of vision $\left(0.391 \mathrm{~mm}^{2}\right)$ was used to perform all the calculations. Cell counting was performed in a blinded manner on coded slides, to avoid bias.

To classify the observed ganglionic organization, the number of PGP 9.5-positive neurons (at least 100 neurons per whole mount, expressed as number of cells $/ \mathrm{mm}^{2}$ ), the size of the neuron body (this term refers to the area of the maximal profile seen in the whole-mount preparations, $\mu \mathrm{m}^{2}$ ), the diameter of the nerve fibers (at least fifty nerve fibers per whole mount, with only primary internodal strands being taken into account, $\mu \mathrm{m}$ ) and the relative ganglionic area (excluding nerve fibers, \%) were semi-automatically measured in ten randomly chosen, non-overlapping microscopic fields per animal. All stained cells were identified as the nerve cells on the basis of their morphology, showing a round or ovoid nucleus and a clear profile.

To quantify the subclasses of myenteric neurons (NOS-positive, ChAT-positive, TH-positive), the positively stained cells were semi-automatically measured in ten randomly chosen, non-overlapping microscopic fields per animal. The stained cells were identified as the nerve cells on the basis of their morphology, showing a round or ovoid nucleus and a clear profile. The final numbers were expressed as the number of neurons per $1 \mathrm{~mm}^{2}$ and as the percentage of the total number of PGP 9.5-positive neurons.

The number of alpha-synuclein-immunopositive cells co-reactive for PGP 9.5 was determined semi-automatically in ten randomly chosen, non-overlapping microscopic fields per animal and expressed as the number of alpha-synuclein-positive neurons per $1 \mathrm{~mm}^{2}$.

The number of Bax-immunopositive neurons in the myenteric area was determined semi-automatically in ten randomly chosen, non-overlapping microscopic fields per animal and expressed as the number of Bax-positive neurons per $1 \mathrm{~mm}^{2}$. 

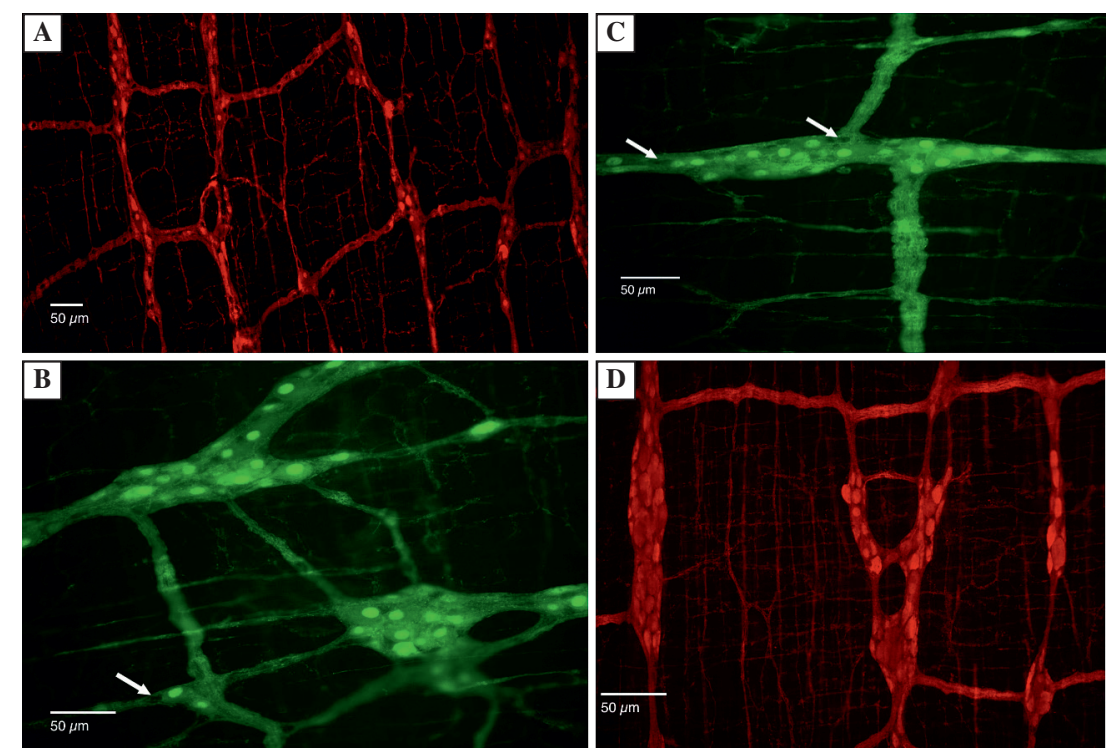

Figure 1. Pan-neuronal marker PGP 9.5 immunoreactivity in the jejunal myenteric plexus. Representative photomicrographs show the immunoreactivity of the pan-neuronal marker PGP 9.5 in the whole mounts of the jejunal myenteric plexus of the salsolinol-treated for two (A, Cy3 staining) or four weeks (B, C, fluorescein staining) and control adult Wistar rats (D, Cy3). Arrows (B, C) indicate abnormal cavities [29] within the ganglia of the jejunal myenteric plexus of rats treated with salsolinol for four weeks. Total magnification: A, $\times 200$; B-D, $\times 400$; scale bar, $50 \mu \mathrm{m}$

Statistical analysis. All data are expressed as the mean and standard deviation (SD). The results were analyzed via one-way analysis of variance (ANOVA), followed by post-hoc tests (Tukey's test) using the STATISTICA 9.0 software package (StatSoft, Tulsa, USA). Statistical significance was set at $\mathrm{p}<0.05$.

\section{Results}

\section{Gross behavior}

None of the salsolinol-treated animals in any of the experiments died or showed any visible disturbances or discomfort to any significant degree. No disturbance of gross motor function was observed in any of the salsolinol-treated rats.

\section{Morphology and morphometry of the jejunal myenteric plexus neurons}

The jejunal myenteric plexus was readily detected in whole-mount preparations stained to detect pan-neuronal marker PGP 9.5. Regardless of the experimental group, the neurons of the myenteric plexus, located between the circular and longitudinal muscle layers, retained their ganglionic organization, showing elongated and triangular arrangements. The ganglia of the myenteric plexuses of control animals were mainly oriented with their long axis parallel to the circular muscle layer. Consistent intense staining of the neuronal somata, nuclei and the majority of neuronal processes was observed (Figure 1D). Most of the neurons were round and formed groups of heterogeneous size.

The whole-mount preparations from the salsolinol-treated groups displayed irregular morphological disruptions in the ganglia of the jejunal myenteric plexus. Ganglia displaying empty areas ("cavities") could be observed [29]. Isolated nerve cells or ganglia with only a small number of nerve cells were observed as well (Figure 1B, C).

The number of myenteric neurons (PGP 9.5-positive), the size of the neuron bodies (Figure 2) and the diameter of the nerve fibers (only primary internodal strands were taken into account) were significantly decreased in both of the salsolinol-treated groups in comparison with the control group (Table 2). Decrease in the population of larger neurons (around $400 \mu \mathrm{m}^{2}$ ) and increase in the population of smaller neurons (around $200 \mu \mathrm{m}^{2}$ ) was observed in salsolinol-treated animals (Figure 2). The ganglionic area (excluding nerve fibers) in the myenteric plexus was reduced by $6 \%$ and $23 \%$ in rats treated with salsolinol for two (S1 group) or four (S2 group) weeks, respectively, in comparison with the control group.

\section{Morphometric analysis of the jejunal myenteric plexus motor neurons}

It was found that ChAT-positive neurons (cholinergic neurons) and NOS-positive neurons (nitrergic 


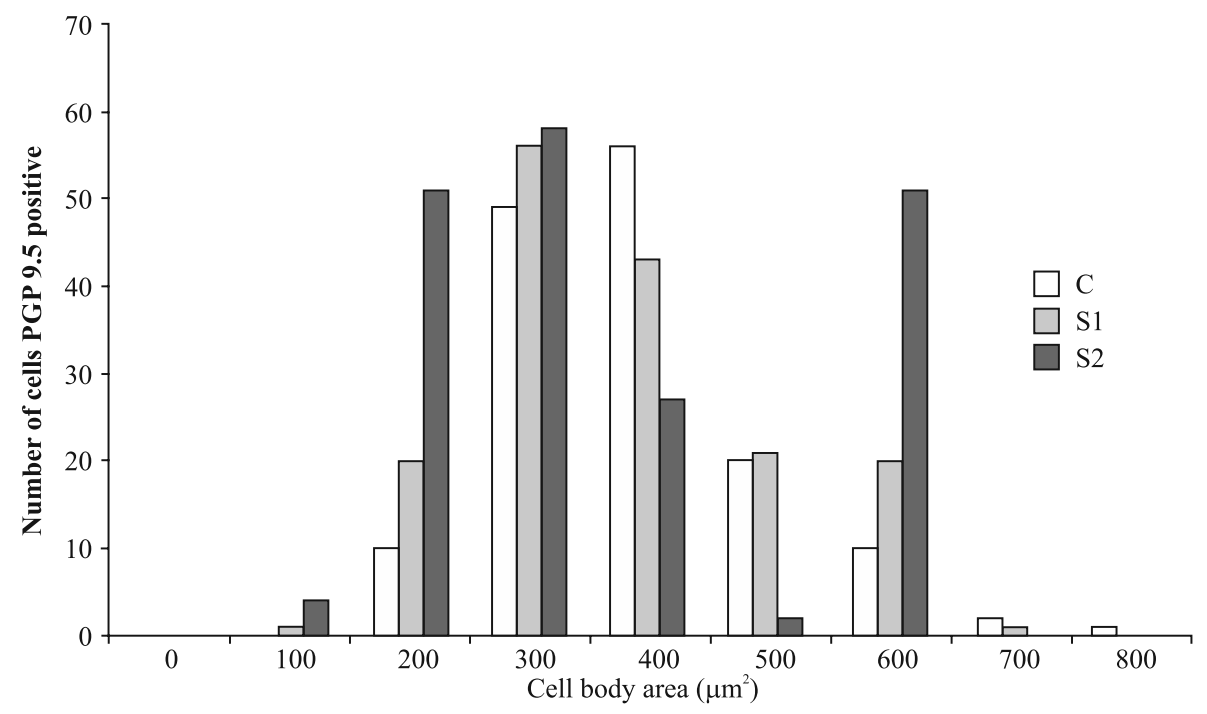

Figure 2. Distributions of the cell body areas of PGP 9.5-immunoreactive neurons in the jejunal myenteric plexus in the salsolinol-treated and control rats. Histogram displaying the distributions of the cell body areas of PGP 9.5-immunoreactive neurons in the jejunal myenteric plexus of the salsolinol-treated for two (S1) or four (S2) weeks and control (C) adult Wistar rats. Each group included 8 animals

Table 2. Morphometric analysis of the myenteric plexus in the jejunum of the salsolinol-treated and control rats

\begin{tabular}{|l|c|c|c|}
\hline \multirow{2}{*}{} & \multicolumn{2}{|c|}{ Groups of rats } \\
\cline { 2 - 4 } & S1 & S2 & C \\
\hline Number of neurons $\left[\right.$ per $\left.\mathrm{mm}^{2}\right]$ & $103.04 \pm 37.5^{*}$ & $103.95 \pm 26.1^{*}$ & $330.55 \pm 98.9$ \\
\hline Neuronal somatic area $\left[\mu \mathrm{m}^{2}\right]$ & $306.32 \pm 104.3^{*}$ & $231.34 \pm 81.8^{*}$ & $17.85 \pm 3.4$ \\
\hline Diameter of nerve fibers $[\mu \mathrm{m}]$ & $16.46 \pm 3.2^{*}$ & $15.74 \pm 3.1^{*}$ & \\
\hline
\end{tabular}

Adult Wistar rats were treated with salsolinol for two (S1) or four (S2) weeks or not treated (C, control). Each group included 8 animals. Data are expressed as the mean $\pm \mathrm{SD}$; *statistically significant vs. control group, $\mathrm{p}<0.05$

neurons) are the two non-overlapping chemical phenotypes that collectively account for almost the entire population of neurons in the myenteric plexus.

The mean number of NOS-positive neurons per $1 \mathrm{~mm}^{2}$ of the jejunal myenteric plexus was significantly lower in the S1 $(\mathrm{p}<0.05)$ and $\mathrm{S} 2(\mathrm{p}<0.001)$ groups than the control group (Figure 3A). Tukey's test showed no differences between the $\mathrm{S} 1$ and $\mathrm{S} 2$ groups. The mean number of ChAT-positive neurons per $1 \mathrm{~mm}^{2}$ of the jejunal myenteric plexus did not differ between any of the experimental groups (Figure 3C).

However, the mean percentage of PGP 9.5-positive myenteric neurons expressing NOS immunoreactivity remained unchanged (Figure 3B), while the mean percentage of PGP 9.5-positive myenteric neurons expressing ChAT immunoreactivity was slightly increased (however, statistically insignificant) in both of the salsolinol-treated group of rats in comparison with the control animals (Figure 3D).

\section{Morphometric analysis of TH-positive neurons in the jejunal myenteric plexus}

Two different monoclonal antisera (Table 1) were used in our experiment to visualize the dopaminergic population of the jejunal myenteric plexus ganglia. However, no cell bodies were visible, with only the dense architecture of the nerve fibers being stained, and no differences were observed between any of the experimental groups.

\section{Alpha-synuclein expression in jejunal myenteric neurons}

The pattern of alpha-synuclein expression in the jejunal myenteric plexus in the salsolinol-treated rats was clearly visible and consistent (Figure 4). A subpopulation of myenteric neurons was immunopositive for alpha-synuclein, with the protein being detected in 

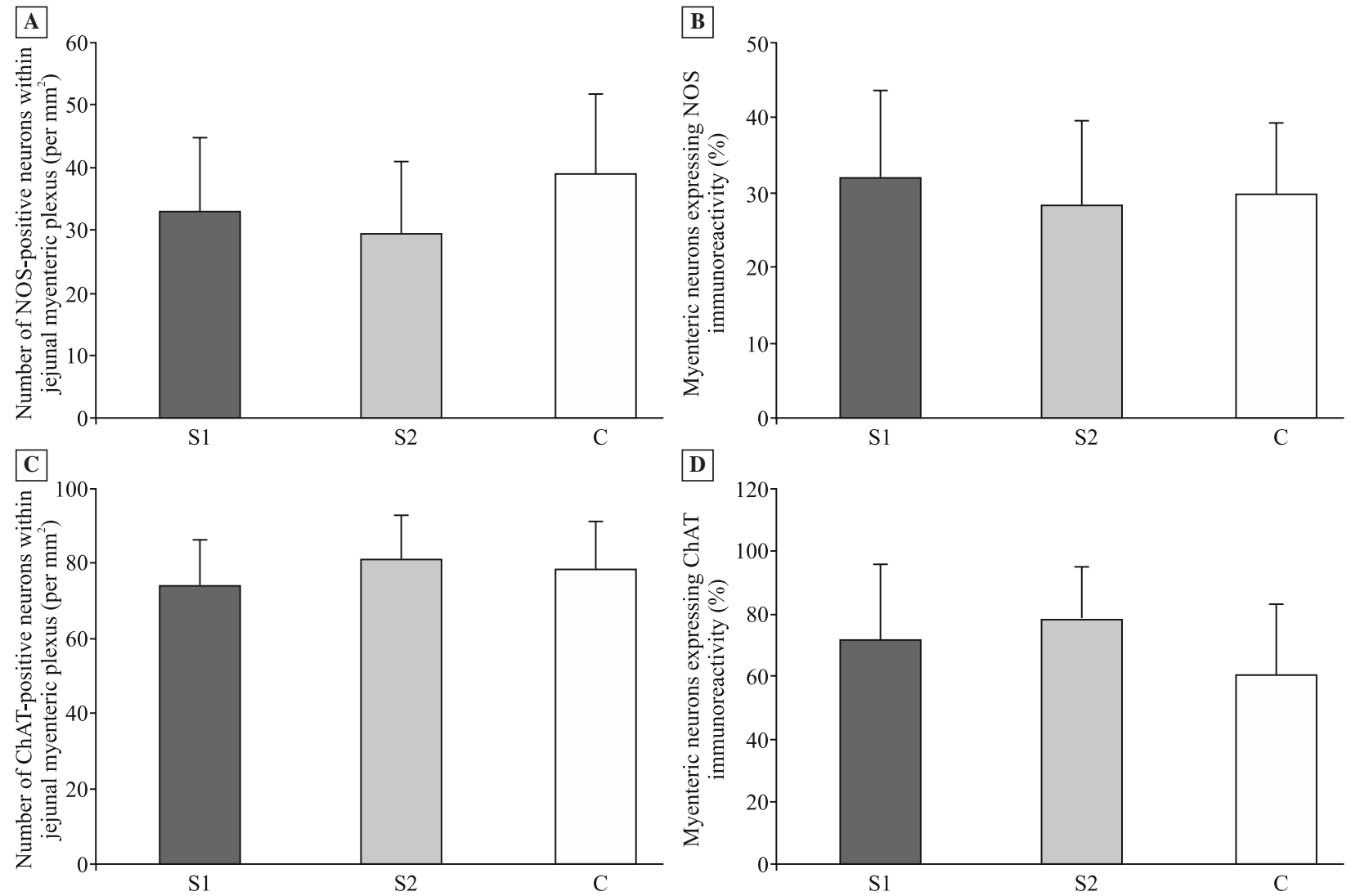

Figure 3. Morphometry of the jejunal myenteric plexus in the salsolinol-treated and control rats. Analysis of the myenteric plexus morphometry in the jejunum of the salsolinol-treated for two (S1) or four (S2) weeks and control (C) adult Wistar rats. Each group included 8 animals. A. Number of NOS-positive neurons within $1 \mathrm{~mm}^{2}$ of the jejunal myenteric plexus; B. Percentage of PGP 9.5-positive myenteric neurons expressing NOS immunoreactivity; C. Number of ChAT-positive neurons within $1 \mathrm{~mm}^{2}$ of the jejunal myenteric plexus; D. Percentage of PGP 9.5-positive myenteric neurons expressing ChAT immunoreactivity. Data are expressed as the mean $\pm \mathrm{SD}$; *statistically significant $v s$. control group, $\mathrm{p}<0.05$

the cytoplasm as well as the nucleus. Alpha-synuclein-positive varicosities occurred within ganglia and encircled individual neurons. Quantitative comparison showed an increase in alpha-positive neurons within $1 \mathrm{~mm}^{2}$ of the jejunal myenteric plexus in the salsolinol-treated rats [S1 $\left(7.433 / \mathrm{mm}^{2} \pm 4.032 ; \mathrm{p}<0.001 ;\right.$ $\mathrm{n}=8)$ and $\left.\mathrm{S} 2\left(6.424 / \mathrm{mm}^{2} \pm 4.081 ; \mathrm{p}<0.001 ; \mathrm{n}=8\right)\right]$ in comparison with control group C $\left(2.661 / \mathrm{mm}^{2} \pm\right.$ $1.764, \mathrm{n}=8$ ). Tukey's test showed no differences between the S1 and S2 groups.

\section{Bax protein expression in jejunal neurons of the myenteric plexus}

Immunohistochemical staining to detect Bax (Figure 5), a pro-apoptotic member of the Bcl-2 family of proteins, revealed a marked increase in the number of Bax-immunopositive cells among myenteric neurons in the $\mathrm{S} 2$ group of rats $\left(1.095 / \mathrm{mm}^{2} \pm 1.82 ; \mathrm{p}<0.05\right.$; $\mathrm{n}=8$ ) in comparison with the control group $C$ $\left(0.256 / \mathrm{mm}^{2} \pm 0.78 ; \mathrm{n}=8\right)$. Tukey's test showed no differences between the S1 $\left(0.894 / \mathrm{mm}^{2} \pm 1.47\right.$; $\mathrm{n}=8$ ) and control or $\mathrm{S} 2$ groups.

\section{Measurement of one-hour stool output}

The mean dry weight of the fecal output of the salsolinol-treated S2 group $(p<0.01)$ was markedly elevated between the $1^{\text {st }}$ and $7^{\text {th }}$ day of the experiment in comparison with the control and the S1 groups (Table 3). The mean dry weight of the fecal output between the $8^{\text {th }}$ and $14^{\text {th }}$ day of the experiment was lower in the salsolinol-treated S2 group in comparison with the control $\mathrm{C} 2$ group and was markedly lower in the $\mathrm{S} 1$ group $(\mathrm{p}<0.05)$ in comparison with the $\mathrm{C} 1$ group (Table 3$)$.

Among the salsolinol-treated groups of rats, the mean stool water content was significantly lower in the S2 group $(p<0.05)$ in comparison with the control $\mathrm{C} 2$ group between the $8^{\text {th }}$ and $14^{\text {th }}$ days and between $15^{\text {th }}$ and $28^{\text {th }}$ days of the experiment (Table 3 ). 

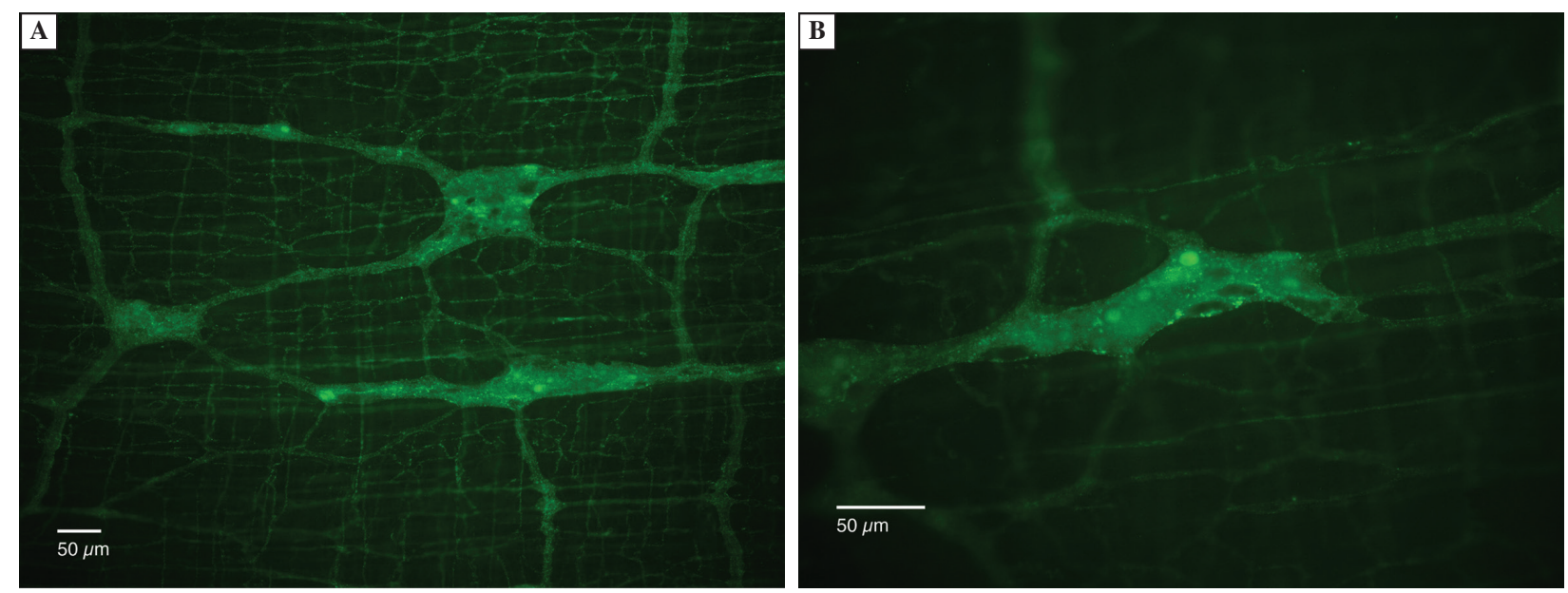

Figure 4. Alpha-synuclein immunoreactivity in the jejunal myenteric plexus. Representative photomicrographs showing the immunoreactivity for alpha-synuclein in the whole mounts of the jejunal myenteric plexus of adult Wistar rats treated with salsolinol for four weeks. Fluorescein staining. Total magnification: A, $\times 200 ; \mathrm{B}, \times 400$; scale bar, $50 \mu \mathrm{m}$
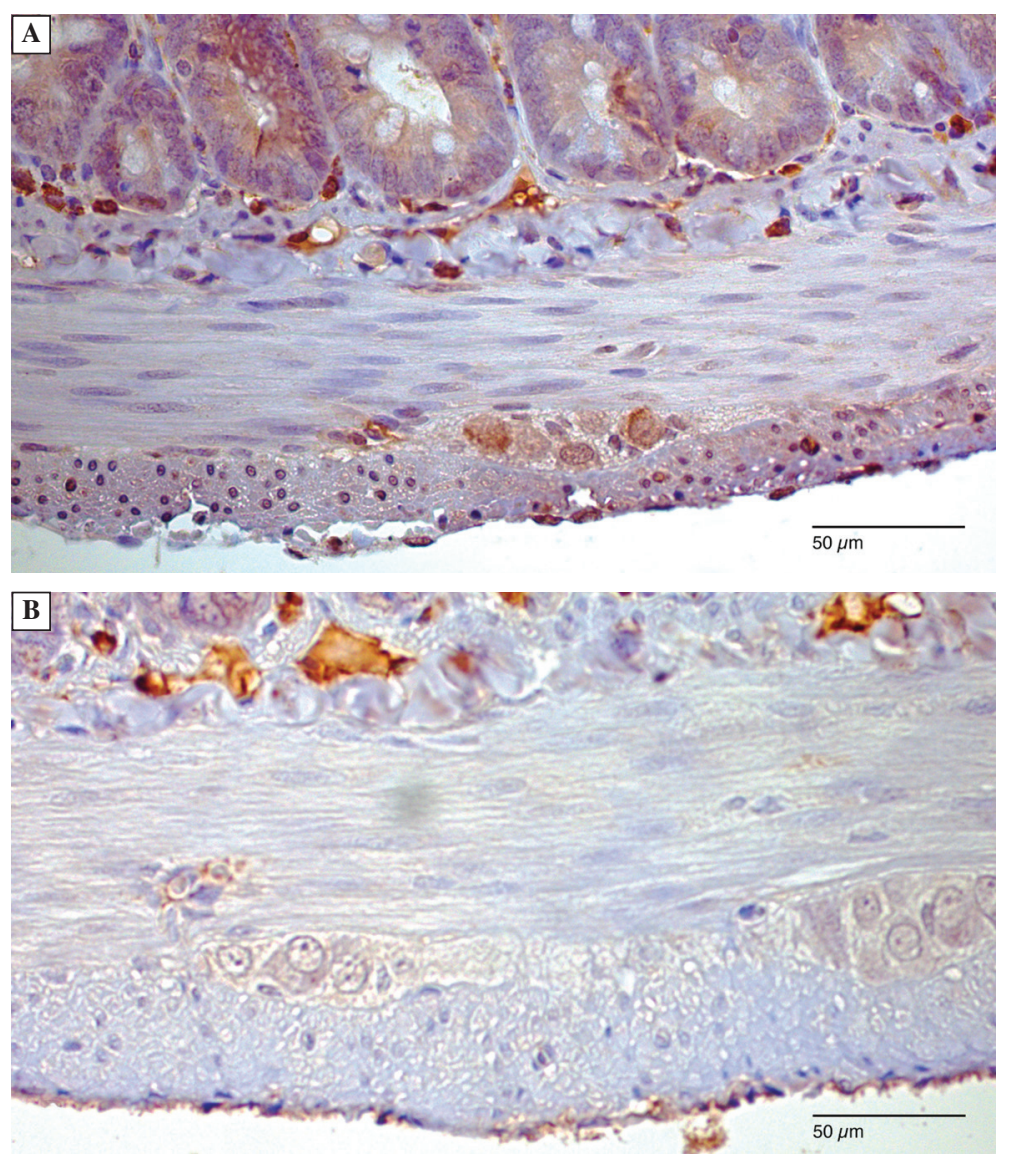

Figure 5. Bax immunoreactivity in the jejunal myenteric plexus. Representative photomicrographs show Bax immunoreactivity for Bax in the jejunal myenteric plexus of adult Wistar rats treated with salsolinol for four weeks (A) and untreated control animals $(\mathbf{B})$. Total magnification: $\times 400$; scale bar, $50 \mu \mathrm{m}$

\section{Measurement of residual solid food in the stomach and gastric emptying}

The amount of food consumed within the one-hour testing period did not differ between the salsolinol- -treated and control groups of rats (Table 4). The percentage of mean residual solid food in the stomachs of the S1 group ( $<<0.05)$ was significantly higher than in the $\mathrm{C} 1$ group (Table 4), but no differences between the $\mathrm{S} 2$ and $\mathrm{C} 2$ groups or the S1 and S2 groups 
Table 3. Cumulative fecal output and stool water content in the salsolinol-treated and control rats

\begin{tabular}{|l|c|c|c|c|}
\hline \multirow{2}{*}{} & \multicolumn{4}{|c|}{ Groups of rats } \\
\cline { 2 - 5 } & S1 & C1 & S2 & C2 \\
\hline${\text { Dry weight }[\mathrm{mg}]^{\mathrm{a}}}^{*}$ & $1.44 \pm 0.51$ & $1.43 \pm 0.29$ & $2.20 \pm 0.31^{*}$ & $1.678 \pm 0.37$ \\
\hline Water $(\%)^{\mathrm{a}}$ & $58.39 \pm 14.54$ & $53.37 \pm 10.64$ & $56.56 \pm 10.98$ & $56.26 \pm 5.51$ \\
\hline Dry weight $[\mathrm{mg}]^{\mathrm{b}}$ & $1.21 \pm 0.37^{*}$ & $1.61 \pm 1.14$ & $0.96 \pm 0.17$ & $1.01 \pm 0.23$ \\
\hline Water $(\%)^{\mathrm{b}}$ & $51.34 \pm 13.47$ & $53.23 \pm 19.72$ & $47.70 \pm 7.22^{*}$ & $59.95 \pm 10.97$ \\
\hline Dry weight $[\mathrm{mg}]^{\mathrm{c}}$ & - & - & $0.84 \pm 0.08$ & $0.82 \pm 0.38$ \\
\hline Water $(\%)^{\mathrm{c}}$ & - & - & $63.96 \pm 5.47^{*}$ & $67.07 \pm 2.84$ \\
\hline
\end{tabular}

Cumulative fecal output (dry weight, $\mathrm{mg}$ ) and stool water content (\%) in adult Wistar rats treated with salsolinol for two (S1) or four weeks (S2) and relevant control animals $(\mathrm{C} 1, \mathrm{C} 2)$, between the (a) $1-7^{\text {th }},(\mathrm{b}) 8-14^{\text {th }}$, and (c) $15-28^{\text {th }}$ days of the experiment. Each group included 8 animals. Data are expressed as the mean $\pm \mathrm{SD}$; *statistically significant $v$. relevant control group, $\mathrm{p}<0.05$

Table 4. Residual solid food in the stomach and gastric emptying in the salsolinol-treated and control rats

\begin{tabular}{|l|c|c|c|c|}
\hline \multirow{2}{*}{} & \multicolumn{3}{|c|}{ Groups of rats } \\
\cline { 2 - 5 } & S1 & C1 & S2 & C2 \\
\hline Food intake during 60 min (g per 1kg of body mass) & $16.4 \pm 3.5$ & $19.6 \pm 2.5$ & $14.8 \pm 6.0$ & $15.4 \pm 2.2$ \\
\hline Residual solid food (\%) & $80.2 \pm 6.1 *$ & $68.4 \pm 6.1$ & $81.3 \pm 4.4$ & $73.6 \pm 6.8$ \\
\hline Water content (\%) & $45.0 \pm 3.4 *$ & $52.0 \pm 3.7$ & $49.3 \pm 4.2$ & $51.8 \pm 3.2$ \\
\hline Gastric emptying after 90 min (\%) & $55.0 \pm 3.1$ & $48.4 \pm 3.0$ & $50.7 \pm 3.8$ & $56.1 \pm 7.1$ \\
\hline
\end{tabular}

Food intake (g per $1 \mathrm{~kg}$ of body mass) during $60 \mathrm{~min}$, residual solid food in the stomach (\%), water content (\%) and gastric emptying after $90 \mathrm{~min}(\%)$ in adult Wistar rats treated with salsolinol for two (S1) or four weeks (S2) and relevant control animals (C1, C2). Each group included 8 animals. Data are expressed as the mean $\pm \mathrm{SD}$; *statistically significant vs. relevant control group, $\mathrm{p}<0.05$

were found. Similarly, the percentage of the gastric emptying between the salsolinol-treated and control rats (Table 4) remained unchanged as well.

The mean water content in the gastric contents was significantly lower in the S1 $(\mathrm{p}<0.05)$ group in comparison with the $\mathrm{C} 1$ group. There were no differences between the $\mathrm{S} 2$ and $\mathrm{C} 2$ or the $\mathrm{S} 1$ and $\mathrm{S} 2$ groups (Table 4).

\section{Measurement of small intestine transit}

The presence of charcoal was recorded in either the $9^{\text {th }}$ segment (corresponding to $90 \%$ transit) or $10^{\text {th }} \mathrm{seg}$ ment (corresponding to $100 \%$ transit) of the intestine in the rats from the four experimental groups (S1, S2, $\mathrm{C} 1, \mathrm{C} 2 ; \mathrm{n}=8$ each). The mean small intestine motility observed in the salsolinol-treated rats did not differ significantly $(\mathrm{S} 1=97.5 \%$; $\mathrm{S} 2=95 \%)$ from control rats $(\mathrm{C} 1=100 \% ; \mathrm{C} 2=97,5 \%)$.

\section{Discussion}

Salsolinol was detected for the first time in urine of Parkinsonian patients administered with L-DOPA in 1970s. This finding stimulated the studies on SAL derivatives in the brain since so far alkaloids had been considered to occur only in plants and had never been considered as a potential pathophysiological factor in PD [20]. The metabolism of salsolinol has been relatively well described $[20,30,31]$. The distribution of the $\mathrm{N}$-methylated and oxidized byproducts of salsolinol likely follow a certain pattern in the human nigrostriatal pathway and should therefore regulate the function of dopaminergic neurons [32]. Thus far, salsolinol and its metabolite N-methyl-salsolinol have been related to the neurodegeneration of central dopaminergic circuits and associated with the pathogenesis of Parkinson disease [19, 20, 33-35]. We decided to investigate the salsolinol treatment as an experimental model in order to mimic gastrointestinal changes observed in patients with Parkinson's disease, in accordance with the Braak's hypothesis.

In the present study, subacute treatment with salsolinol caused a destructive effect on the myenteric neurons since number of PGP 9.5-positive neurons, the size of the neuron bodies and the diameter of the nerve fibers were significantly decreased in both of the salsolinol-treated groups in comparison with the control group. Decrease in the population of larger neurons (around $400 \mu \mathrm{m}^{2}$ ) 
and increase in the population of smaller neurons (around $200 \mu \mathrm{m}^{2}$ ) was observed in salsolinol-treated animals as well. The ganglionic area in the myenteric plexus was also reduced in the salsolinoltreated animals. Conversely, unilateral treatment with 6-hydroxydopamine did not affect the total number of HuC/D-immunopositive (human neuronal protein) myenteric neurons in the proximal and distal segments of the ileum and colon of rats according to Blandini et al. [16].

Myenteric neuron shrinkage together with an increased number of myenteric neurons expressing Bax protein observed in our experiment might also suggest that the observed effect was mediated through the induction of apoptotic pathways. Evidence for the role of apoptotic cell death in PD arises from morphological and molecular studies in cell cultures, animal models, as well as human studies on postmortem brains from Parkinsonian patients [36, 37]. Bax is a primary effector of cell death that translocates from the cytosol to the mitochondria upon stress, where it facilitates cytochrome c release and the subsequent caspase cascade activation [38]. Some experiments showed that Bax is an important regulator of apoptotic neurodegeneration and that Bax plays a pivotal role in the destruction of dopamine-producing neurons in a mouse model of PD [39]. In MPTP (1-methyl-4-phenyl-1,2,3,6-tetrahydropyridine)-injected mice Bax expression levels were increased in regions specifically affected by the neurodegeneration processes $[39,40]$. So far, Naoi et al. demonstrated the cell death caused by salsolinol in human dopaminergic neuroblastoma SH-SY5Y cells to be apoptotic [18]. Apoptosis caused by this neurotoxin is thought to be mediated by an intracellular sequential process, loss of mitochondrial membrane potential, activation of caspases and DNA fragmentation [18, 41].

According to functional classification, enteric motor neurons are either inhibitory (e.g. NOS-positive neurons) or excitatory (e.g. ChAT-positive neurons). The guinea pig has become a model species for identifying these neuron types in the enteric nervous system [42]. However, there are differences in quality and quantity of enteric neurons between different species. This heterogeneity is further related to the thickness of the gut wall or the amount of connective tissue present. Different factors influence the quality of whole-mount preparations and the penetration of antibodies, resulting in different staining intensities [43].

In our experiment, we observed a statistically significant decrease in the number of NOS-positive (nitrergic) neurons in the jejunal myenteric plexus. This finding is consistent with the results of 6-hydroxy- dopamine treatments, which reduced the number of nitrergic neurons in the distal ileum and proximal colon in rodents [16]. The average integrated optical densities of nNOS-positive areas in the gastric antrum and colon tissue are decreased in rats with unilateral 6-hydroxydopamine lesions [44]. However, treatment through either intraperitoneal injection of MPTP [45] or continuous subcutaneous administration of rotenone [25] was found to cause no alterations in the numbers or morphology of nitrergic neurons. Thus, our data might suggest that salsolinol exerts stronger toxic effect on nitrergic neurons than MPTP or rotenone.

Furthermore, salsolinol caused no alterations in jejunal ChAT-positive (cholinergic) neuron numbers. Similarly, no changes in cholinergic neurons were observed in rats with brain unilateral 6-hydroxydopamine lesions [44], and there were no differences in the density of these neurons found in the MPTP [45] or rotenone [25] models.

Treatment with intraperitoneal injections of MPTP has been reported to cause a $40 \%$ reduction of the number of dopaminergic neurons in the ENS of mice, and a relaxation defect in the colon of these animals was confirmed by the electrophysiological recordings [45]. Unilateral 6-hydroxydopamine lesions increase the average integrated optical densities of $\mathrm{TH}$-positive areas in the gastric antrum and colon tissue of rats [44]. In our experiment, we did not observe any TH-positive neurons (cell bodies) localized within the jejunal myenteric plexus. However, a dense network of TH-positive fibers was clearly visible. In fact, there is no direct evidence that dopaminergic neurodegeneration should play a fundamental role in ENS neurodegeneration. Rather, intrinsic inhibitory neural pathways are expected to be critical for the gastrointestinal dysfunctions observed in Parkinsonian patients.

Rotenone administered subcutaneously with osmotic pumps induces a delay in gastric emptying and a transient decrease in stool frequency [25]. Rats with unilateral 6-hydroxydopamine lesions in the nigrostriatal dopaminergic neurons display marked inhibition of the propulsive activity. Blandini et al. suggested that this inhibition is a consequence of the impairment of nitric oxide-mediated descending inhibition during peristalsis [46]. In the same model, Zhu et al. reported a decrease in the weight and water content of the fecal matter and an increase in the percentage of residual solid food. Lesioned rats showed delayed gastric emptying and constipation [44]. In the present study, we noted a decrease in transit in the large intestine. Furthermore, there was a notable decrease in water content, especially in 
rats treated with salsolinol for four week, which might be associated with the loss of NOS-positive neurons. However, we did not detect any disturbances in small intestine transit. The salsolinol-treated animals also presented reduced gastric emptying and decreased water content of the gastric residues.

Gastrointestinal symptoms are prominent and disabling manifestations of Parkinson's disease [7, 46-48]. Some such manifestations, such as constipation, may precede somatic motor symptoms by many years [4]. Neuropathologic studies consistently demonstrate the involvement of the enteric nervous system [9, 49-52] and the dorsal motor nucleus of the vagus (DMV) based on alpha-synuclein histopathology [53, 54]. These symptoms are classified as drooling, pharyngeal or esophageal dysmotility, early satiety and nausea, constipation and defecatory dysfunction, as elegantly summarized in a review by Cerosimo and Benaroch [55]. Chronic constipation most likely reflects a loss of neurons in the myenteric plexus $[56,57]$ and may also occur as a manifestation of the age-related neurodegeneration of the ENS [58]. Thus, reduced colonic transit is likely to reflect the involvement of myenteric neurons in PD [9, 50]. Siddiqui et al. confirmed that autonomic dysfunction occurs in PD patients at a greater frequency and with a greater severity than in comparably aged controls [59]. It appears that gastrointestinal dysfunction might be a direct consequence of enteric neuron pathology and neurotransmitter impairment $[8,9,59]$ or an indirect consequence of the pathology of the substantia nigra and striatum $[60,61]$. Several observations reported to date support the well-known Braak hypothesis [10, 62-64]. This pathology is expected to start in the gastric submucosal plexus [9] and spread to the central nervous system via axons of the DMV [11].

There are parallels between brain and enteric neurons. Pathology induced by alpha-synuclein analogous to that described in the CNS has been demonstrated in the gut. The formation of such aggregates is believed to depend on the presence of either genetically abnormal proteins or normal proteins that undergo a change in structure due to being present in excess, exceeding their clearance ability [65, 66]. Alpha-synuclein-positive inclusions form Lewy bodies which were detected in the myenteric plexuses of PD patients, particularly within enteric inhibitory motor neurons [9, 17, 50, 67]. These inclusions have also been detected in non-symptomatic subjects with PD-related brain lesions limited to the lower brainstem, thereby supporting the hypothesis that the gastrointestinal tract may be an early target of the disease [11]. In our experiment, salsolinol induced alpha-synuclein aggregation within the myenteric neurons. Similar accumulation was observed after 6-hydroxydopamine administration [68].

The presence of alpha-synuclein inclusions in VIP-positive [51] and NOS-positive neurons suggests that reduced transit in the large intestine may result from disturbed smooth muscle relaxation due to the loss of inhibitory motor neurons $[16,44]$. Therefore, both the constipation and the delay gastric emptying observed in PD patients are expected to be caused by impaired descending inhibition, mediated by nitric oxide during peristalsis. Impairment of enteric nitrergic neurons could induce over-contraction of smooth muscle, leading to muscle spasm and slow colon motility, increasing water absorption from intestinal contents $[44,69]$. Interestingly, we also observed a decrease in body weight gain and slower adipose tissue accumulation in the salsolinol-treated animals, which may result from abnormal gastrointestinal motility and absorption [70].

In conclusion, our results confirm the role of inhibitory motor neurons in the pathogenesis of gastrointestinal dysfunctions, similar to the findings of other experimental studies. These results also suggest that endogenous neurotoxins, such as salsolinol, might lead to the accumulation of alpha-synuclein aggregates in the ENS neurons and may be useful in experiments elucidating the pathomechanisms of the neurodegenerative processes.

\section{Acknowledgments}

The research project reported in this manuscript has been founded by the Jagiellonian University Medical College grants K/ZDS/002513 and K/DSC/000261.

\section{References}

1. Wood JD, Alpers DH, Andrews PL. Fundamentals of neurogastroenterology. Gut. 1999;45:II6-II16. PMID: 10457039.

2. Furness JB. The enteric nervous system: normal functions and enteric neuropathies. Neurogastroenterol Motil. 2008;20:32-38. doi: 10.1111/j.1365-2982.2008.01094.x.

3. Konturek PC, Brzozowski T, Konturek SJ. Stress and the gut: pathophysiology, clinical consequences, diagnostic approach and treatment options. J Physiol Pharmacol.2011;62:591-599. PMID: 22314561.

4. Abbott RD, Petrovitch H, White LR et al. Frequency of bowel movements and the future risk of Parkinson's disease. Neurology. 2001;57:456-462. PMID: 11502913.

5. Probst A, Bloch A, Tolnay M. New insights into the pathology of Parkinson's disease: does the peripheral autonomic system become central? Eur J Neurol. 2008;15:1-4. doi: 10.1111/j. 1468-1331.2008.02057.x.

6. Jost WH. Autonomic dysfunctions in idiopathic Parkinson's disease. J Neurol. 2003;250:I28-I30. PMID: 12761632.

7. Pfeiffer RF. Gastrointestinal dysfunction in Parkinson's disease. Lancet Neurol. 2003;2:69-73. PMID: 12849267.

8. Braak H, Rüb U, Gai WP, Del Tredici K. Idiopathic Parkinson's disease: possible routes by which vulnerable neuronal 
types may be subject to neuroinvasion by an unknown pathogen. J Neural Transm. 2003;110:517-536. PMID: 12721813.

9. Braak H, de Vos R a I, Bohl J, Del Tredici K. Gastric alpha-synuclein immunoreactive inclusions in Meissner's and Auerbach's plexuses in cases staged for Parkinson's disease-related brain pathology. Neurosci Lett. 2006;396:67-72. PMID: 16330147.

10. Hawkes CH, Del Tredici K, Braak H. A timeline for Parkinson's disease. Parkinsonism Relat Disord. 2010;16:79-84. doi: 10.1016/j.parkreldis.2009.08.007.

11. Hawkes $\mathrm{CH}$, Del Tredici K, Braak H. Parkinson's disease: a dual-hit hypothesis. Neuropathol Appl Neurobiol.2007;33:599_ -614. PMID: 17961138.

12. McDowell K, Chesselet M-F. Animal models of the non-motor features of Parkinson's disease. Neurobiol Dis. 2012;46: 597-606. doi: 10.1016/j.nbd.2011.12.040.

13. Gerlach M, Riederer P. Animal models of Parkinson's disease: an empirical comparison with the phenomenology of the disease in man. J Neural Transm. 1996;103:987-1041. PMID: 9013391.

14. Meredith GE, Sonsalla PK, Chesselet M. Animal models of Parkinson's disease progression. Acta Neuropathol. 2008;115:385-398. doi: 10.1007/s00401-008-0350-x.

15. Nagatsu T. Parkinson's disease: changes in apoptosis-related factors suggesting possible gene therapy. J Neural Transm. 2002;109:731-745. PMID: 12111464.

16. Blandini F, Balestra B, Levandis $\mathrm{G}$ et al. Functional and neurochemical changes of the gastrointestinal tract in a rodent model of Parkinson's disease. Neurosci Lett. 2009;467:203-207. doi: 10.1016/j.neulet.2009.10.035.

17. Phillips RJ, Walter GC, Wilder SL, BaronowskyEA, PowleyTL. Alpha-synuclein-immunopositive myenteric neurons and vagal preganglionic terminals: autonomic pathway implicated in Parkinson's disease? Neuroscience. 2008;153:733-750. doi: 10.1016/j.neuroscience.2008.02.074.

18. Naoi M, Maruyama W, Akao Y, Zhang J, Parvez H. Apoptosis induced by an endogenous neurotoxin, $\mathrm{N}$-methyl(R)salsolinol, in dopamine neurons. Toxicology. 2000;153:123-141. PMID: 11090952.

19. Naoi M, Maruyama W, Dostert P et al. Dopamine-derived endogenous $1(\mathrm{R}), 2(\mathrm{~N})$-dimethyl-6,7-dihydroxy-1,2,3,4tetrahydroisoquinoline, N-methyl-(R)-salsolinol, induced parkinsonism in rat: biochemical, pathological and behavioral studies. Brain Res. 1996;709:285-295. PMID: 8833765.

20. Naoi M, Maruyama W, Nagy GM. Dopamine-derived salsolinol derivatives as endogenous monoamine oxidase inhibitors: occurrence, metabolism and function in human brains. Neurotoxicology. 2004;25:193-204. PMID: 14697894.

21. Greene JG, Dingledine R, Greenamyre JT. Neuron-selective changes in RNA transcripts related to energy metabolism in toxic models of parkinsonism in rodents. Neurobiol Dis. 2010;38:476-481. doi: 10.1016/j.nbd.2010.03.014.

22. Fornai F, Schlüter OM, Lenzi P et al. Parkinson-like syndrome induced by continuous MPTP infusion: convergent roles of the ubiquitin-proteasome system and alpha-synuclein. Proc Natl Acad Sci USA. 2005;102:3413-3418. PMID: 15716361.

23. Alvarez-Fischer D, Guerreiro S, Hunot $\mathrm{S}$ et al. Modelling Parkinson-like neurodegeneration via osmotic minipump delivery of MPTP and probenecid. J Neurochem. 2008;107:701-711. doi: 10.1111/j.1471-4159.2008.05651.x.

24. Li ZS, Schmauss C, Cuenca A, Ratcliffe E, Gershon MD. Physiological modulation of intestinal motility by enteric dopaminergic neurons and the D2 receptor: analysis of dopamine receptor expression, location, development, and function in wild-type and knock-out mice. J Neurosci. 2006; 26:2798-2807. PMID: 16525059.

25. Greene JG, Noorian AR, Srinivasan S. Delayed gastric emptying and enteric nervous system dysfunction in the rotenone model of Parkinson's disease. Exp Neurol. 2009;218:154-161. doi: 10.1016/j.expneurol.2009.04.023.

26. Anitha M, Gondha C, Sutliff R et al. GDNF rescues hyperglycemia-induced diabetic enteric neuropathy through activation of the PI3K/Akt pathway. J Clin Invest. 2006;116:344-356. PMID: 16453021.

27. Stefanini M, De Martino C, Zamboni L. Fixation of ejaculated spermatozoa for electron microscopy. Nature. 1967; 216:173-174. PMID: 4862079.

28. Pasternak A, Gajda M, Gil K et al. Evidence of interstitial Cajal-like cells in human gallbladder. Folia Histochem Cytobiol. 2012;50:581-585. doi: 10.5603/19673.

29. Hanani M, Fellig Y, Udassin R, Freund HR. Age-related changes in the morphology of the myenteric plexus of the human colon. Auton Neurosci. 2004;113:71-78. PMID: 15296797.

30. Maruyama W, Strolin-Benedetti M, Naoi M. N-methyl(R) salsolinol and a neutral $\mathrm{N}$-methyltransferase as pathogenic factors in Parkinson's disease. Neurobiology. 2000;8:55-68. PMID: 11008878.

31. Maruyama W, Dostert P, Naoi M. Dopamine-derived 1-methyl-6,7-dihydroxyisoquinolines as hydroxyl radical promoters and scavengers in the rat brain: in vivo and in vitro studies. J Neurochem. 1995;64:2635-2643. PMID: 7760044.

32. Maruyama W, Nakahara D, Ota M et al. N-methylation of dopamine-derived 6,7-dihydroxy-1,2,3,4-tetrahydroisoquinoline, (R)-salsolinol, in rat brains: in vivo microdialysis study. J Neurochem. 1992;59:395-400. PMID: 1629715.

33. Maruyama W, Abe T, Tohgi H, Naoi M. An endogenous MPTP-like dopaminergic neurotoxin, N-methyl(R)salsoli$\mathrm{nol}$, in the cerebrospinal fluid decreases with progression of Parkinson's disease. Neurosci Lett. 1999;262:13-16. PMID: 10076861.

34. Antkiewicz-Michaluk L. Endogenous risk factors in Parkinson's disease: dopamine and tetrahydroisoquinolines. Pol J Pharmacol. 2002;54:567-572. PMID: 12866710.

35. Antkiewicz-Michaluk L, Michaluk J, Romańska I, Papla I, Vetulani J. Antidopaminergic effects of 1,2,3,4-tetrahydroisoquinoline and salsolinol. J Neural Transm. 2000;7:1009-1019. PMID: 11041279

36. Honig LS, Rosenberg RN. Apoptosis and neurologic disease. Am J Med. 2000;108:317-330. PMID: 11014725.

37. Lev N, Melamed E, Offen D. Apoptosis and Parkinson's disease. Prog Neuropsychopharmacol Biol Psychiatry. 2003;27:245-250. PMID: 12657363.

38. Jürgensmeier JM, Xie Z, Deveraux Q et al. Bax directly induces release of cytochrome $\mathrm{c}$ from isolated mitochondria. Proc Natl Acad Sci USA. 1998;95:4997-5002. PMID: 9560217.

39. Vila M, Jackson-Lewis V, Vukosavic S et al. Bax ablation prevents dopaminergic neurodegeneration in the 1-methyl-4-phenyl-1,2,3,6-tetrahydropyridine mouse model of Parkinson's disease. Proc Natl Acad Sci USA. 2001;98:2837-2842. PMID: 11226327.

40. Charan RA, Johnson BN, ZaganelliS, NardozziJD, LaVoie MJ. Inhibition of apoptotic Bax translocation to the mitochondria is a central function of parkin. Cell Death Dis. 2014;5:e1313. doi: 10.1038/cddis.2014.278.

41. Maruyama W, Boulton AA, Davis BA, Dostert P, Naoi M. Enantio-specific induction of apoptosis by an endogenous neurotoxin, $\mathrm{N}$-methyl(R)salsolinol, in dopaminergic $\mathrm{SH}$ -SY5Y cells: suppression of apoptosis by N-(2-heptyl)-N- 
-methylpropargylamine. J Neural Transm. 2001;108:11-24. PMID: 11261742.

42. Furness JB. Types of neurons in the enteric nervous system. J Auton Nerv Syst. 2000;81:87-96. PMID: 10869706.

43. Beck M, Schlabrakowski A, Schrödl F, Neuhuber W, Brehmer A. ChAT and NOS in human myenteric neurons: co-existence and co-absence. Cell Tiss Res. 2009;338:37-51. doi: 10.1007/ /s00441-009-0852-4.

44. Zhu HC, Zhao J, Luo CY, Li QQ. Gastrointestinal dysfunction in a Parkinson's disease rat model and the changes of dopaminergic, nitric oxidergic, and cholinergic neurotransmitters in myenteric plexus. $J$ Mol Neurosci. 2012;47:15-25. doi: 10.1007/s12031-011-9560-0.

45. Anderson G, Noorian AR, Taylor G et al. Loss of enteric dopaminergic neurons and associated changes in colon motility in an MPTP mouse model of Parkinson's disease. Exp Neurol. 2007;207:4-12. PMID: 17586496.

46. Blandini F, Armentero M-T, Martignoni E. The 6-hydroxydopamine model: news from the past. Parkinsonism Relat Disord. 2008;14:S124-S129. doi: 10.1016/j.parkreldis.2008.04.015.

47. Edwards LL, Quigley EM, Harned RK, Hofman R, Pfeiffer RF. Characterization of swallowing and defecation in Parkinson's disease. Am J Gastroenterol. 1994;89:15-25. PMID: 8273792.

48. Edwards LL, Quigley EM, Pfeiffer RF. Gastrointestinal dysfunction in Parkinson's disease: frequency and pathophysiology. Neurology. 1992;42:726-732. PMID: 1565224.

49. Wakabayashi K, Takahashi H. Neuropathology of autonomic nervous system in Parkinson's disease. Eur Neurol. 1997;38:2-7. PMID: 9387796.

50. Wakabayashi K, Takahashi H, Takeda S, Ohama E, Ikuta F. Lewy bodies in the enteric nervous system in Parkinson's disease. Arch Histol Cytol. 1989;52:191-194. PMID: 2554944.

51. Wakabayashi K, Takahashi H, Ohama E, Ikuta F. Parkinson's disease: an immunohistochemical study of Lewy body-containing neurons in the enteric nervous system. Acta Neuropathol. 1990;79: 581-583. PMID: 1972853.

52. Banach T,ZurowskiD, Gil K, Krygowska-Wajs A, Marszałek A, Thor PJ. Peripheral mechanisms of intestinal dysmotility in rats with salsolinol induced experimental Parkinson's disease. J Physiol Pharmacol. 2006;57:291-300. PMID: 16845232.

53. Del Tredici K, Duda JE. Peripheral Lewy body pathology in Parkinson's disease and incidental Lewy body disease: four cases. J Neurol Sci. 2011;310:100-106. doi: 10.1016/j.jns. 2011.06.003

54. Del Tredici K, Rüb U, De Vos RAI, Bohl JRE, Braak H. Where does Parkinson disease pathology begin in the brain? J Neuropathol Exp Neurol. 2002;61:413-426. PMID: 12030260.

55. Cersosimo MG, Benarroch EE. Neural control of the gastrointestinal tract: implications for Parkinson disease. Mov Disord. 2008;23:1065-1075. doi: 10.1002/mds.22051.

56. Cersosimo MG, Benarroch EE. Pathological correlates of gastrointestinal dysfunction in Parkinson's disease. Neurobiol Dis. 2012;46:559-564. doi: 10.1016/j.nbd.2011.10.014.
57. Wedel T, Spiegler J, Soellner S et al. Enteric nerves and interstitial cells of Cajal are altered in patients with slow-transit constipation and megacolon. Gastroenterology. 2002;123:1459-1467. PMID: 12404220.

58. Camilleri M, Cowen T, Koch TR. Enteric neurodegeneration in ageing. Neurogastroenterol Motil. 2008;20:418-429. doi: 10.1111/j.1365-2982.2008.01134.x.

59. Siddiqui MF, Rast S, Lynn MJ, Auchus AP, Pfeiffer RF. Autonomic dysfunction in Parkinson's disease: a comprehensive symptom survey. Parkinsonism Relat Disord. 2002;8:277-284. PMID: 12039423.

60. Pouclet H, Lebouvier T, Coron E et al. A comparison between colonic submucosa and mucosa to detect Lewy pathology in Parkinson's disease. Neurogastroenterol Motil. 2012;45:305-309. doi: 10.1111/j.1365-2982.2012.01887.x.

61. Colucci M, Cervio M, Faniglione Met al. Intestinal dysmotility and enteric neurochemical changes in a Parkinson's disease rat model. Auton Neurosci. 2012;169:77-86. doi: 10.1016/ j.autneu.2012.04.005.

62. Kalaitzakis ME, Graeber MB, Gentleman SM, Pearce RKB. The dorsal motor nucleus of the vagus is not an obligatory trigger site of Parkinson's disease: a critical analysis of alpha-synuclein staging. Neuropathol Appl Neurobiol. 2008;34:284-295. PMID: 18053026.

63. Braak H, Tredici K, Rüb U. Staging of brain pathology related to sporadic Parkinson's disease. Neurobiol Aging. 2003;24:197-211. PMID: 12498954.

64. Dickson DW, Fujishiro H, DelleDonne A et al. Evidence that incidental Lewy body disease is pre-symptomatic Parkinson's disease. Acta Neuropathol. 2008;115:437-444. doi: 10.1007/ /s00401-008-0345-7.

65. Kingsbury A, Bandopadhyay R, Silveira-Moriyama L et al. Brain stem pathology in Parkinson's disease: an evaluation of the Braak staging model. Movement Disord. 2010;25:2508-2515. doi: $10.1002 / \mathrm{mds} .23305$.

66. Lee HJ, Suk JE, Lee KW. Transmission of synucleinopathies in the enteric nervous system of A53T alpha-synuclein transgenic mice. Exp Neurobiol. 2011;20:181-188. PMID: 22355263.

67. Natale G, Pasquali L, Paparelli A, Fornai F. Parallel manifestations of neuropathologies in the enteric and central nervous systems. Neurogastroenterol Motil. 2011;23:1056-1065. doi: 10.1111/j.1365-2982.2011.01794.x.

68. Colucci M, De Giorgio R, Blandini F et al. Enteric nitrergic neuron defect and gut dysfunction in a rat model of Parkinson's disease. Gastroenterology. 2009;136:A-154. doi: 10.1016/S0016-5085(09)60695-8.

69. Drolet RE, Cannon JR, Montero L, Greenamyre JT. Chronic rotenone exposure reproduces Parkinson's disease gastrointestinal neuropathology. Neurobiol Dis. 2009;36:96-102. doi: 10.1016/j.nbd.2009.06.017.

70. Kurnik M, Gil K, Bugajski A, Bujak-Giżycka B, Madej J, Thor P. The influence of salsolinol on basic rat metabolism. Fol Med Cracov. 2012;52:5-20. PMID: 24852683.

Submitted: 24 November, 2014

Accepted after reviews: 24 March, 2015 Available as AoP: 26 March, 2015 